\title{
APPLICATION OF TRIZ THEORY IN REDUCING NOISE OF FUEL
}

\section{PUMP}

\author{
Mengqi Ge', Jun Zhou' ${ }^{2}$ \\ ${ }^{I}$ School of Mechanical Engineering, Shanghai University of Engineering Science, Shanghai, China \\ ${ }^{2}$ School of Mechanical Engineering, Shanghai University of Engineering Science, Shanghai, China
}

\begin{abstract}
In view of the vibration and noise generated by the operation of a turbo-type fuel pump, the TRIZ theory is proposed to improve the vibration and noise of the fuel pump. First of all, the function analysis of TRIZ is used to find out the causes of vibration and noise of turbo-type fuel pump. Then the standard solutions of TRIZ theory are obtained by using the tools of material-field analysis and contradictory matrix in TRIZ theory. The proposal in which a fuel inlet passage of a fuel pump is optimized can be used to reduce the flow pulsation; In addition, changing the turbine fuel pump impeller material can also reduce the friction, thereby reducing vibration and noise. By comparative experiments, the improved method can effectively improve the vibration of the fuel pump and reduce the noise of the fuel pump.
\end{abstract}

Keywords: TRIZ theory, functional analysis, fuel pump, noise

\section{INTRODUCTION}

With the development and progress of the times, people travel more and more depending on the car as a means of transportation. In recent years, the world automotive market has been expanding. Both the output and the demand have continued to grow, making the car an indispensable part of people's lives Important items. When people want to buy a car, they tend to pay more attention to car comfort rather than just pursue car performance. A problem of the fuel pump noise is becoming noticeable, attaching great importance by the car designers. Considering the vibration and noise of the fuel pump, literature[1] studied the effects of the commutator on the noise of the fuel pump and proposed a method in which the material of the commutator was changed to reduce the noise of the fuel pump. Literature[2]studied how the impeller distribution of the turbo-type fuel pump affected the vibration and noise of the pump, and made a proposal in which the isometric impeller distribution was changed into a random distribution of uneven impeller. In addition, a numerical simulation method for computing fluid dynamics was used to verify the effectiveness of the improved structure. verifies. Literature[3] studied the effect of flow on the noise of the fuel pump, and proposed a variable flow fuel pump to reduce the noise. Using the flow rate proportional to the load of the engine, the noise of the fuel pump can be improved. Literature[4] studied the effects of fuel supply tank structure onfuel pump noise and proposed the design of grooves and protrusions on the bottom of the mailbox to improve the fuel noise.

The above research on vibration and noise of fuel pump relies heavily on the designer's experience and there is no systematic solving process. But TRIZ theory has its own tools and database, which is an innovative subject that leads to people's innovation step by step by dedicated tools rather than imagines[5] blindly and randomly. Starting from relative knowledge of TRIZ theory. This paper first analyzes the causes of turbine fuel pump noise by using the function analysis in TRIZ theory. Then the problem of noise of turbo pump is solved by using object-field analysis model and contradiction matrix. Then, combined with the actual status, an exact solution of solving the fuel pump noise problem is obtained.

\section{THE OVERALL FRAMEWORK OF INNOVATIVE DESIGN BASED ON TRIZ THEORY}

TRIZ theoretical system is a subject which first applies a series of analytical tools such as functional analysis of technical systems, contradictions analysis, resource analysis and object-field models to analyse the standard invention problems, and then the problem can be addressed by using standard solutions, principle of technological contradiction and innovation, the principle of physical contradiction of separation and the effect of knowledge base and other problem-solving tools. A nonstandard problem can be easily solved by using the invention of problem-solving algorithm tools[6]. As a result, a complex problem solving process with uncertainties is transformed into a systematic scientific operation process.

The basic idea of using TRIZ theory to solve practical problems is: first, transform the actual problem into the standard problem of TRIZ; then use the TRIZ theory to derive the standard solution. Three steps are included[7]: 
[1]. Through functional analysis, resource analysis and contradiction analysis, we can directly use effect knowledge base to find high-quality solutions.

[2]. Through object-field analysis and contradiction matrix analysis, if it is a standard problem, the solution can be obtained from 76 standard solutions and 40 invention principles.

[3]. If it is a non-standard invention problem, then Ariz(Algorithm for Inventive-Problem Solving) can be used to analyze and describe the problem. If it can not be solved at a time, the description should be performed repeatedly, so as to accurately describe the problem and define the contradiction, and the actual problem can be transformed to a standard problem. And then, a high solution can be achieved by using 76 standard solutions, 40 invention principles and effects knowledge base[8].

The process of solving the turbo pump noise based on TRIZ theory is shown in Fig-1.

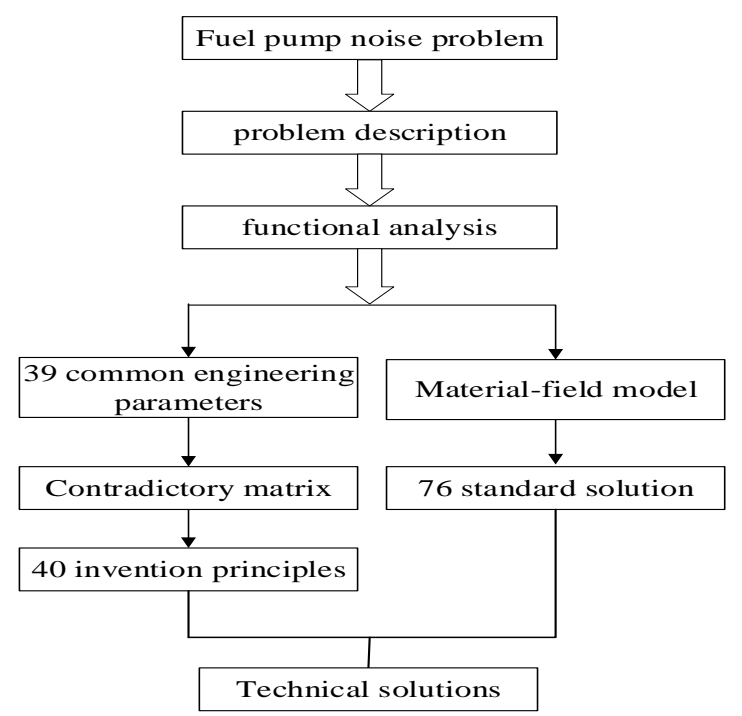

Fig-1: The process of solving the noise of turbine fuel pump based on TRIZ Theory

\section{THE ANALYSIS OF TURBINE FUEL PUMP VIBRATION AND NOISE CAUSES}

Its working principle is: the fuel pump motor is energized, and the impeller on the shaft is driven by the motor to rotate. Under the action of centrifugal force, the fuel flows into the suction chamber of the pump body and flows out of the discharge chamber of the pump body[9]. Due to the constant decrease of the fuel in the suction chamber, a vacuum will be formed in the suction chamber and the fuel in the fuel tank will be sucked in from the oil inlet. At the same time, the amount of fuel discharged from the chamber will increase and the fuel pressure will increase. When a certain pressure is reached, the outlet valve will be propped up and fuel will be inhaled from the outlet[10]. The outlet valve will prevent the fuel back too the fuel tank when the fuel stops working, thereby maintaining the loop with a certain pressure to facilitate the nextstart of the fuel pump.

Turbofan pump function is to ensure that a sufficient number of fuel can be delivered from the fuel tank to the injection pump, and to ensure that there is a certain supply pressure to overcome various resistance in the pipeline so that the fuel can also be delivered in the low-pressure pipeline fuel[11]. The main structure of the fuel pump includes turbo pump body, motor, housing, filter, pressure relief valve and check valve and other components. If regarding the entire fuel pump work process as a system, then a subsystem which completes and affects its subfunction includes turbo pump body, impeller, filter, oil channel, the super system for the mailbox, the rectangular box that the system, Super system, subsystem structure, as shown in Fig-2. Itcan be seen that, the pump body, impeller, oil channel are key factors of the fuel pump vibration and noise.

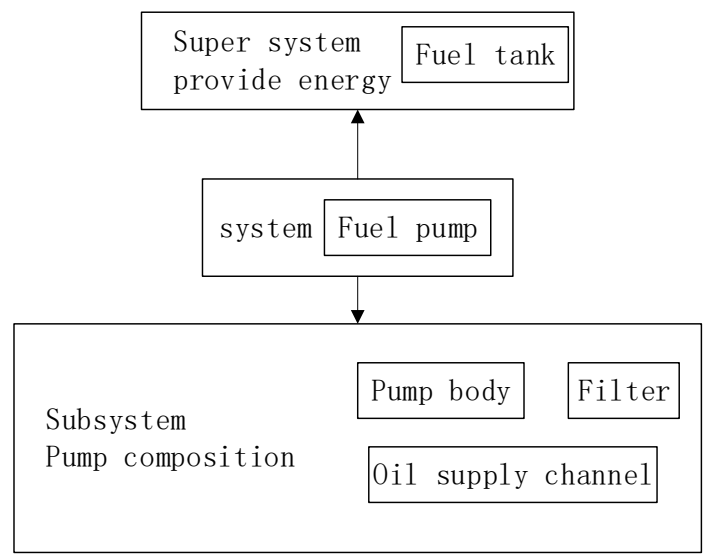

Fig 2: turbo fuel pump function analysis

Through the analysis of the functional components of TRIZ theory, it can be seen that there are two sources of noise for the turbo-type fuel pumps, noise generated by pump operation and noise generated by high-pressure fuel injection[12].

The noise generated by the pump core operation is:

Reason One: the fuel is pumped into the pump chamber through the inlet, and then is sent out from the pump outlet of the pump housing. The turbine noise will be directly scattered to the outside following the inlet channel, resulting in the entire turbine pump pressure level increased and large noise.

The noise generated by high pressure fuel shocks is:

Reason Two: the blade structure of the impeller of the existing turbo-type fuel pump is evenly distributed. Therefore, when the fuel pump is in a high-speed operation environment, the impeller blades have the same oil output frequency and high-frequency fluid noise. In addition, the existing impeller structure can not meet the pressure of the application environment due to the pressure of the increasing application environment of fuel. Because at high 
pressure and high speed operation, the impeller cannot be automatically suspended in the oil, so the impeller can only be tightly along the wall of the fuel pump chamber running at high speed, prolonged operation will cause the fuel pump body wear, thereby increasing Large fuel pump noise.

The frequent use of turbo pumps leads to increasing rotation resistance between the rotor shaft and bearing and increasing surface wear. As a result, the speed of the electric fuel pump is reduced, the flow performance is reduced, the current is increased, and the noise is increased. These reasons will cause turbo pump noise, but the reason one and reason two are mainly analyzed by using TRIZ theory.

\section{APPLICATION OF TRIZ THEORY IN} REDUCING TURBINE PUMP NOISE

Through the functional analysis of the components of the turbo-type fuel pump, we can draw the conclusion that the turbo-type fuel pump produces noise during operation, and then use the main tools of TRIZ theory to reduce the noise of the turbo pump efficiently.

\subsection{Object-Field Model Analysis}

For the noise generated in reason one the application of TRIZ theory of material - field tools for analysis, the construction of material - field analysis model, shown in Fig-3. Among them: S1 is the inlet pipeline, S2 is the fuel, and F1 is the pressure field. Noise is generated due to the impact of the fuel in the inlet pipe, and then spreads through the oil inlet channel. Since S1 is detrimental to the noise produced by $\mathrm{S} 2$, the solution to the problem is solved using the apodization of subdivision object field model 1.2 of the 76 standard solutions. See Table 1 for the standard solutions it contains.

According to the standard solution S1.2.1 introduction of off-the-shelf substance S3 to eliminate the harmful effects of enlightenment, you can install the damping pump shock absorber, the use of diaphragm and leaf spring to absorb pressure pulsation, the fuel pipeline pulsating pressure Weaken, and reduce noise.

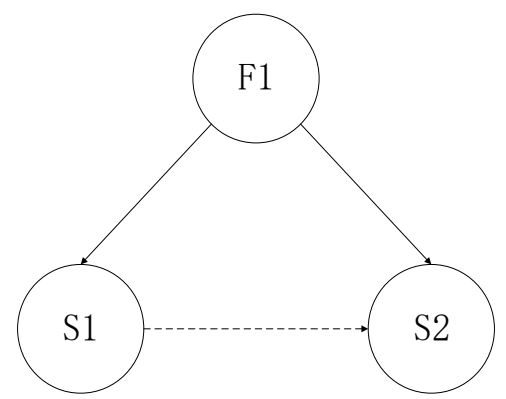

Fig-3: Material - field analysis model

\begin{tabular}{|c|c|}
\hline Subclass & Standard solution \\
\hline \multirow{5}{*}{$\begin{array}{l}\text { S1.2 } \\
\text { Dismantlin } \\
\mathrm{g} \\
\text { objects } \\
\quad \text { field }\end{array}$} & $\begin{array}{l}\text { S1.2.1Introduce readily available } \\
\text { substances }\end{array}$ \\
\hline & $\begin{array}{l}\text { S1.2.2 The introduction of existing } \\
\text { S1(S2) deformation (or variant) }\end{array}$ \\
\hline & $\begin{array}{l}\text { S1.2.3 The substance S3 is } \\
\text { introduced inside (or outside) the } \\
\text { existing substance S1 (or S2) }\end{array}$ \\
\hline & S1.2.4 Introduce field F2 \\
\hline & $\begin{array}{l}\text { S1.2.5 Demagnetization or the } \\
\text { introduction of the opposite magnetic } \\
\text { field }\end{array}$ \\
\hline
\end{tabular}

According to the standard solution in Table 1 S1.2.3 The introduction of substance S3 in the interior (or exterior) of the existing object $\mathrm{S} 1$ (or S2) to eliminate the harmful effects suggests that the interior of the oil inlet may be corrugated with corrugated piping because textured pipe structure can effectively reduce the pressure pulsation of oil, thereby reducing the noise when the fuel is pumped into the oil pump, so as to achieve the purpose of reducing vibration and noise.

In addition, the diameter of the oil inlet passage is gradually reduced from the end remote from the pump chamber to the bent portion. This will help accelerate the fuel. Before and after the improvement diagram shown in Fig-4 and Fig-5.

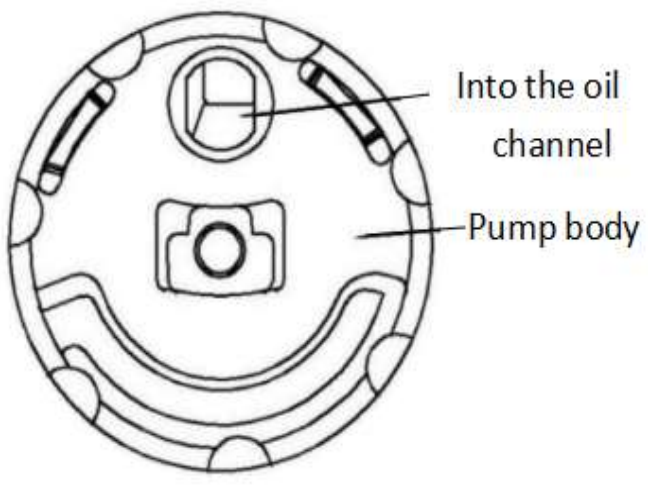

Fig-4: Covering before improving

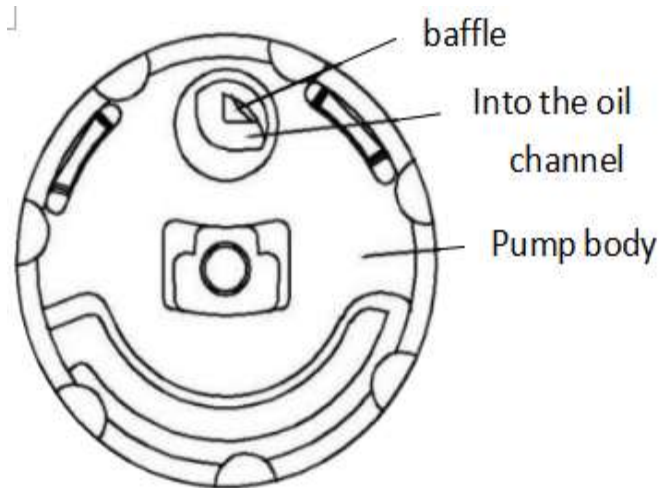

Fig-5: Covering after improving

\subsection{Analysis with Contradictory Matrix}


For the noise generated in reason two the cause is the structure of the impeller, so the engineering parameters that need to be improved are 13: Shape and Engineering Parameter 23: Material Loss while the Deteriorating Engineering Parameter is 36 . System Complexity Sex.

Put the project parameters into Archiechurch matrix, the solution set is: $1,10,16,24,28,29,35$. Innovative solutions based on the principle of innovation are shown in Table 2.

Table 2: Original understanding of innovation

\begin{tabular}{|c|c|c|}
\hline principle & $\begin{array}{l}\text { The original } \\
\text { solution }\end{array}$ & $\begin{array}{l}\text { The original solution to the } \\
\text { application method }\end{array}$ \\
\hline 1 & $\begin{array}{l}\text { Separation } \\
\text { method }\end{array}$ & $\begin{array}{l}\text { An object can be divided } \\
\text { into several independent } \\
\text { parts; the object is designed } \\
\text { to } \\
\text { reorganizationand } \\
\text { disassemble of the part. }\end{array}$ \\
\hline 10 & $\begin{array}{l}\text { Pre-acting } \\
\text { method }\end{array}$ & $\begin{array}{l}\text { Refers to the } \\
\text { implementation of an event } \\
\text { before an event, so that they } \\
\text { proceed. }\end{array}$ \\
\hline 16 & $\begin{array}{l}\text { Part beyond } \\
\text { the law }\end{array}$ & $\begin{array}{l}\text { When the desired effect is } \\
\text { difficult to achieve } 100 \% \text {, it } \\
\text { should achieve a slightly } \\
\text { greater or less than the ideal } \\
\text { effect, thus simplifying the } \\
\text { problem. }\end{array}$ \\
\hline 24 & $\begin{array}{l}\text { Intermediar } \\
\text { y method }\end{array}$ & $\begin{array}{l}\text { Refers to the use of some } \\
\text { medium in the middle of a } \\
\text { number of vectors for } \\
\text { innovative inventions; such } \\
\text { as: the use of migration can } \\
\text { have intermediate role or } \\
\text { transfer; a(easy toseparate) } \\
\text { object temporarily attached } \\
\text { to another object. }\end{array}$ \\
\hline 28 & $\begin{array}{l}\text { Mechanical } \\
\text { system } \\
\text { replacement } \\
\text { method } \\
\end{array}$ & $\begin{array}{l}\text { Refers to the use of physics } \\
\text { or other forms of role to } \\
\text { replace the mechanical } \\
\text { system. }\end{array}$ \\
\hline 29 & $\begin{array}{l}\text { Pressure } \\
\text { method }\end{array}$ & $\begin{array}{l}\text { Refers to the use of gas or } \\
\text { liquid instead of the fixed } \\
\text { part of the object. Such as } \\
\text { inflatable or fluid filled } \\
\text { structures, air cushions, } \\
\text { hydrostatic and } \\
\text { hydrodynamic structures. }\end{array}$ \\
\hline 35 & $\begin{array}{l}\text { Performanc } \\
\text { e } \\
\text { conversion } \\
\text { method }\end{array}$ & $\begin{array}{l}\text { Refers to changing the } \\
\text { attributes of a system to } \\
\text { provide a useful innovation. }\end{array}$ \\
\hline
\end{tabular}

Through systematic analysis of the problem, the principle solution $1,10,16,28,29,35$ are not suitable, you can get some inspiration through the original solution 24 intermediary method. Using the intermediary method, we can slightly modify the structure of the impeller. First of all, to change the uniform distribution of the impeller blades, the association of the impeller is set around the annular blade groove, but the blade groove is not evenly distributed in the blade. The blade is placed between the outer ring and the inner ring of the blade groove, and the blade is inclined and symmetrical to each other in the direction of oil inlet and outlet of the blade groove. In addition, since the impeller can not be automatically suspended in the oil underhighspeed and high-pressure conditions, a deflector floating tank with a pressure balance hole is provided in the direction of oil feeding to the impeller.

Through systematic analysis of the problem, the principle solution 1,10,16,28,29,35 are not suitable, you can get some inspiration through the original solution 24 intermediary method. Using the intermediary method, we can slightly modify the structure of the impeller. First of all, to change the uniform distribution of the impeller blades, the association of the impeller is set around the annular blade groove, but the blade groove is not evenly distributed in the blade. The blade is placed between the outer ring and the inner ring of the blade groove, and the blade is inclined and symmetrical to each other in the direction of oil inlet and outlet of the blade groove. In addition, since the impeller can not be automatically suspended in the oil under highspeed and high-pressure conditions, a deflector floating tank with a pressure balance hole is provided in the direction of oil feeding to the impeller.

As the impeller produces feed points during production, a second pressure balance hole has to be provided in the float tank. The advantage of this structure is that the fuel can flow from the high pressure zone to the low pressure zone through the set balance hole during the operation of the fuel pump. The centrifugal force generated by the impeller at high speed enables the fuel to be uniformly distributed on the low pressure surface of the impeller to reduce the noise. Under special circumstances, for example, when the pressure difference is relatively large, the fuel in the float tank can also lubricate, and the friction between the impeller and the inner wall of the pump chamber becomes smaller, thereby reducing the wear and reducing the noise. The before and after Optimized impeller are shown in Fig-6 and Fig-7.

The enlightenment obtained by the pressure method is that because the pressure of the oil pump operates at high speed, a cavity is formed between the impeller and the pump body, and the friction between the impeller and the oil pump is great. When the compressor runs for a long time, frictional noise will be generated. Inspired by the pressure method, the impeller can be made of nylon material, thereby reducing friction noise. 


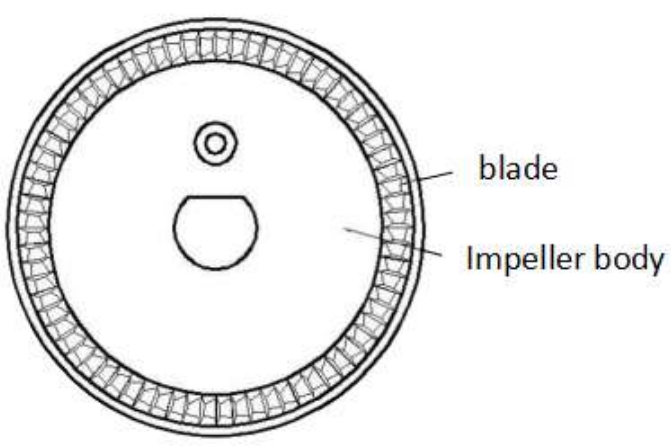

Fig 6: impeller before optimizing

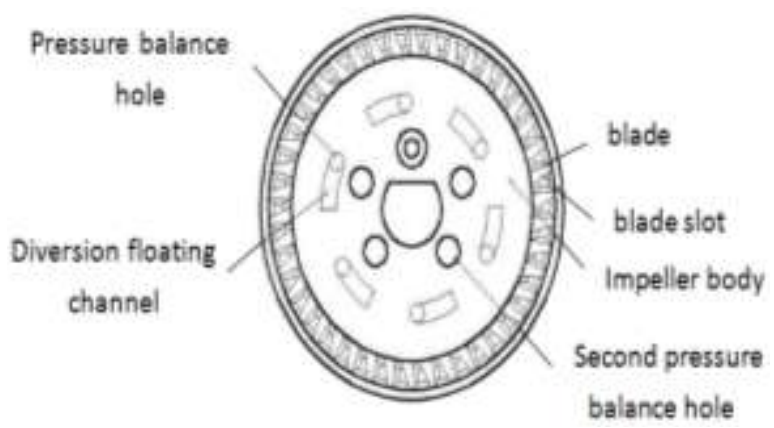

Fig 7: impeller after optimizing

\subsection{Optimization Test}

The general solution as above is obtained by TRIZ theory, and the obtained solution set is optimized on the fuel pump. The experimental test is used to verify that the noise of the optimized fuel pump is obviously reduced, and the feasibility of TRIZ theory is obtained.

The verification experiment needs to be carried out in a semi-anechoic chamber, and the required instruments include a data acquisition system, a microphone, an acceleration sensor, a stand, a DC power supply and the like. The test bench is arranged as shown in Figure 8, with the fuel tank on the bracket with the acceleration sensor placed on the upper surface of the fuel pump and a microphone hanging at $30 \mathrm{~cm}$ from the upper surface of the tank[13]. And then add fuel in the mailbox, and open the DC power supply switch until the fuel pump works smoothly, and through the data acquisition system to save the 10 s of the noise audio, and through matlab to deal with the audio and obtain the noise spectrum, repeat the above Figure 9 shows the spectrum of the fuel pump before optimization, and Figure 10 shows the spectrum of the noise after optimization. From the spectrum before and after optimization, it can be seen that the amplitude of the optimized spectrum is significantly reduced and the spectrum is more stable. This shows that the noise after optimization has obviously improved.

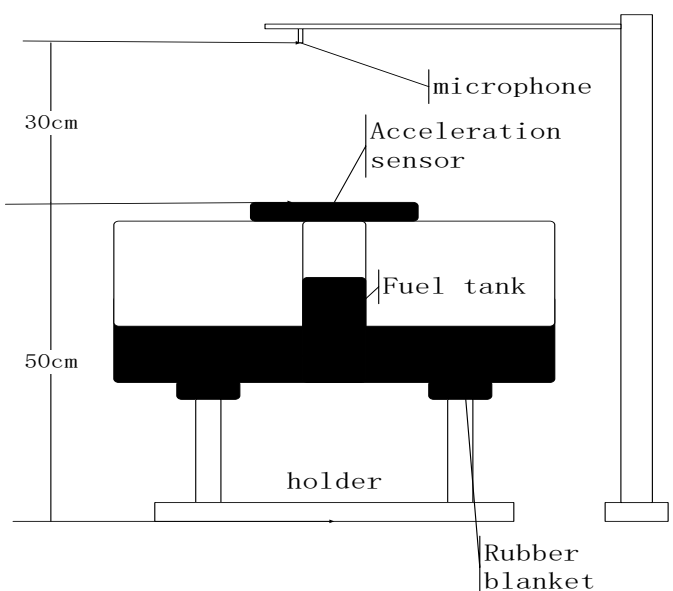

Fig 8: noise test bench layout

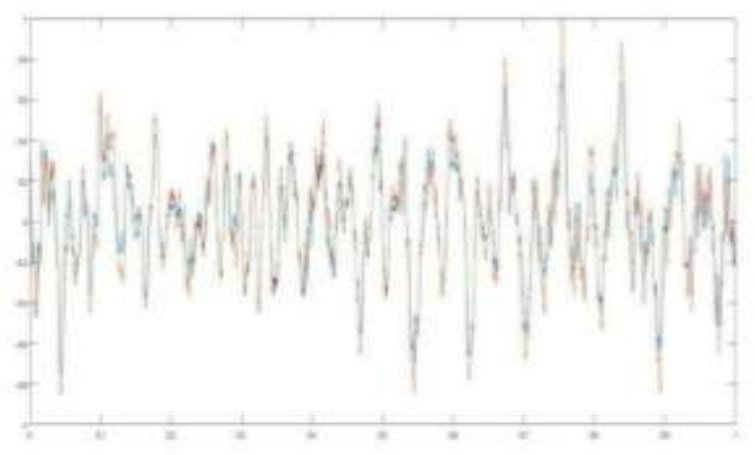

Fig 9: The amplitude of the fuel Pump noise before optimizing

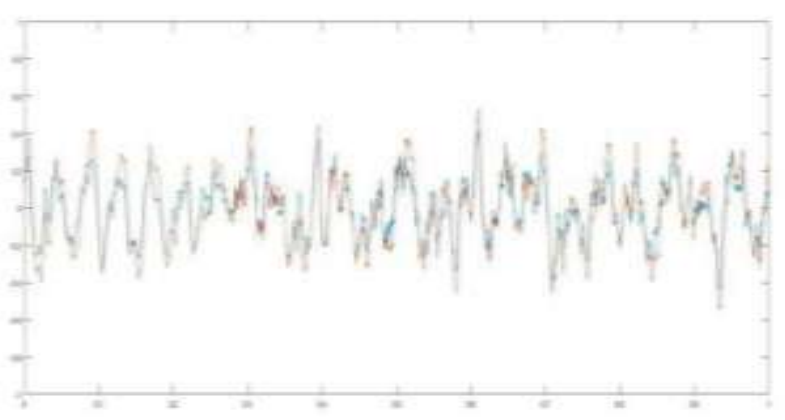

Fig 10: The amplitude of the fuel pump noise after optimizing

\section{CONCLUSION}

It is difficult to grasp the essence of the problem by using the traditional brainstorming method, and it takes a lot of time and effort. By using TRIZ theory innovation method, starting directly from the essence of the problem of technical conflict and using the conflict matrix to narrow the scope , can play a multiplier effect[14]. Therefore, this paper uses TRIZ theory and component functional analysis to find out the causes of fuel pump noise. Through transforming into engineering problems, using object-field analysis tools and contradictory matrix in TRIZ theory, we can get the ideal solution. Relating to practice, we can get the specific 
solution. It proves that TRIZ theory plays a very important role in practical application. However, there are still some problems in TRIZ theory. The most important one is that its problem analysis has no systematic process and requires a great deal of knowledge as a foundation. And there is no set of systematic approach for turning practical problems into engineering problems that turncan be solved by using TRIZ theory.

\section{REFERENCES}

[1]. Wang Jiawei. Automotive fuel pump impeller structure of the impact of noise[D].Southwest Jiaotong University, 2014.

[2]. Meng Shuaiqi. Gasoline gasoline supply system noise control $[\mathrm{J}]$. Science and Technology Innovation and Application, 2016 (12): 48-49.

[3]. Zou, Liu Bing.Analysis of automotive fuel system gas resistance and noise generation and prevention[J] science and technology economy, 2016 (22): 41.

[4]. Su Shirong, Zhou Wei, Wan Yinsheng.Optimization of idling noise of automobile fuel pump[J]. Journal of Xihua University (Natural ScienceEdition), 2017,36 (06): 47-50.

[5]. Chen Xiaojun. Automotive fuel pump noise analysis and optimization[A].China Society of Automotive Engineers. 2010 China Society of Automotive Engineers Conference Paper[C].China Society of Automotive Engineers, 2010: 5.

[6]. The sustainable future of hydro power: A critical analysis of cooling units via the Theory of Invention Problem Solving and life Cycle Assessment methods[J]. Journalof cleanerProduction, 2017,142: 2446-2453.

[7]. Product Design Using Theory of innovation and Problem Solving TRIZ Technology for Induction Machine[C]. 7th International Conference On Intelligent Systems And Control, Coimbatore: ISCO,2013: 44-48.

[8]. TANG Zhi-rong.Research on Innovative Design of Mechanical Products Basedon TRIZ[D].Tianjin:Tianjin University of Technology, 2011.

[9]. Pan Chengyi, Jiang Jingang. TRIZ theory and innovative design methods[M].Beijing:Tsinghua University Press. 2015.

[10]. Shen Menghong .TRIZ theory and practice of mechanical innovation [M].Beijing:Mechanical Industry Press, 2012.

[11]. Song Jin Gui, Li Dong translation. Direct injection gasoline engine technology[M].Beijing:Mechanical Industry Press .2012.

[12]. WANG Zeng.Design Design of DC Fuel Pump Based on TRIZ Theory[J].Journal of Mechanical Design,2016, 33 (11): 105-109.

[13]. Zhang Junfeng. A roller vane pump noise reduction technology [D]. Lanzhou:Lanzhou University of Technology, 2014.

[14]. Mu Haining, Jin Yanfang. Noise Analysis and Optimization of a Car Oil Transfer Pump[J].Agricultural Equipment and Vehicles Engineering,2015,53 (5): 68-71. 